Case Report

\title{
Neglected Penile Constricting Injury in a 9 Year Old Child; a Case Report and Challenges of Management
}

\section{Muzzammil Abdullahi, Bashir Yunusa, Sharfuddeen Abbas Mashi, Sani Ali Aji", Sani Usman Alhassan}

Urology Unit, Department of Surgey, Bayero University, Aminu Kano Teaching Hospital, Kano, Nigeria

\author{
Email address \\ saniaji2004@yahoo.com (S. A. Aji)
}

\section{To cite this article:}

Muzzammil Abdullahi, Bashir Yunusa, Sharfuddeen Abbas Mashi, Sani Ali Aji, Sani Usman Alhassan. Neglected Penile Constricting Injury in a 9 Year Old Child; a Case Report and Challenges of Management. Journal of Surgery. Vol. 4, No. 1, 2016, pp. 1-3.

doi: $10.11648 /$ j.js.20160401.11

\begin{abstract}
Penile constricting injury is a rare urologic emergency which if not treated early can lead to various vascular and mechanical complications. We present a case of neglected penile constriction in a 9 year old boy and the methods we used in removing the constricting object.
\end{abstract}

Keywords: Penile Constricting Injury, Management, Challenges

\section{Introduction}

Penile constriction is a rare urologic emergency and often presents as a challenging clinical condition that will require urgent removal of the constricting agent in order to prevent gangrene of the penis. Several types of objects predominantly metallic and plastic rings are used for different reasons to constrict the penis. It generally follows self placement of a constricting device for enhancing sexual stimulation or by persons suffering from psychiatric illness. In children, it usually follows childish experimentation and where it is applied to control enuresis or urinary incontinence [1,2].

Constriction of the penis by an encircling object leads to swelling of the penis distal to the object because of the initial blockage of the venous return. This may result in massive enlargement of the penis due to lymph oedema. The blockade may involve arterial supply and after several hours penile strangulation can result in ischemic necrosis and gangrene of the penis. In the former, the necrosis may result from anoxia associated with venous stasis. In addition, various degree of mechanical penile injuries are recognized such as skin ulceration, urethral injuries, constriction of corpus spongiosum and corpora cavernosa, development of urethral fistula, and loss of distal penile sensations [3].

The presence of constricting penile device causes considerable anxiety to the patient or his parents because of fear of loss of the organ. Presentation may be due to penile pain, acute urinary retention, ulcers or outright gangrene of the penis [1].

Several methods have been used for successful removal of the constricting object. These include string method and it's modification, use of nut cutter and hack saws, penile aspiration and excision of penile skin and Buck's fascia. The choice of the method for removal depends on the type and size of the metal object, the incarceration time, trauma grade, and availability of equipment $[1,4]$.

We present a case of neglected penile constriction by a metallic nut in a 9 year old child who presented 72 hours after insertion of the nut. We also share our experience with the various approaches used in removing the constricting object.

\section{Case Report}

A 9 year old primary school pupil was brought to accident and emergency unit of our hospital by his father with complains of penile pain and worsening difficulty in passing urine 6 hours prior to presentation. He was said to have inserted the metallic nut by himself until it reaches the root of the penis 72 hours prior. He made all efforts to remove it but was not successful. He did not inform his parents for fear of being beaten by them until when he started having penile pain and difficulty in passing urine. However, he was not in acute urinary retention and there was no history of bleeding from the urethra or any part of the penis. There was no fever or 
vomiting.

He is the $9^{\text {th }}$ child of his mother in a polygamous setting of 2 wives. Father is a petty trader and mother is a full term housewife.

No history of psychiatric illness in the child or the family.

When examined, he was calm, not in painful distress, not febrile (axillary temperature; 36.70c), not pale. He was fully conscious and alert with Glasgow coma score (GCS) of 15. Pulse and blood pressure were within normal range. Examination of the external genitalia revealed a thick metallic nut constricting the root of the penis with grossly oedematous distal penis and some blisters at the corona (Fig. 1 \&2).

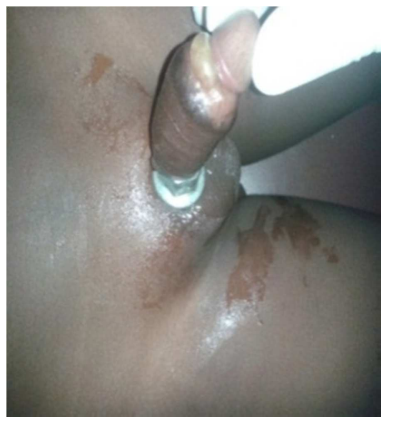

Figure 1. Penile constricting metallic nut (side view). Note the tight nut and blister.

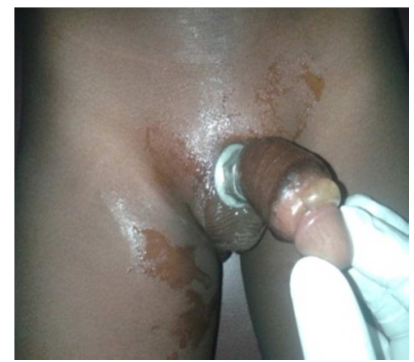

Figure 2. Penile constricting metallic note (anterior view).

The scrotum and its contents were normal.

He had urgent complete blood count and serum urea, electrolytes and creatinine which were all within the normal limits.

He was prepared and taken to the theatre for removal of the nut. Under general anaesthesia (GA), warm compressus was initially applied severally with the aim of reducing the oedema but there was no significant reduction. This was followed by bilateral cavernosal aspiration using 20G cannula. Significant reduction of the swelling of the distal penis was achieved. However attempt to remove the nut by string method after adequate lubrication failed. At this point we decided to deglove the penile skin and gently squeeze the skin out of the nut proximally (Fig. 3\&4) The nut was then gradually milked out with adequate lubrication. The phallus was copiously irrigated with normal saline, an $8 \mathrm{G}$ siliconized urethral catheter was passed which drained clear amber coloured urine and the skin was then reconstructed over the phallus and dressed with antibiotic gauze (Fig. 5).

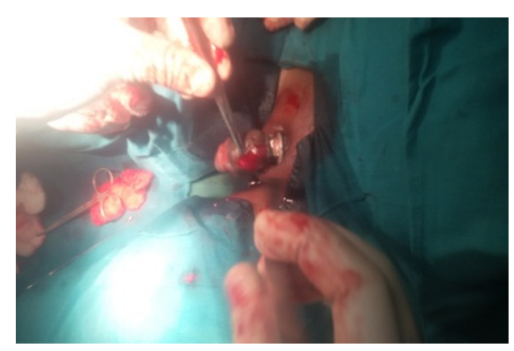

Figure 3. Penis being degloved with nut in place.

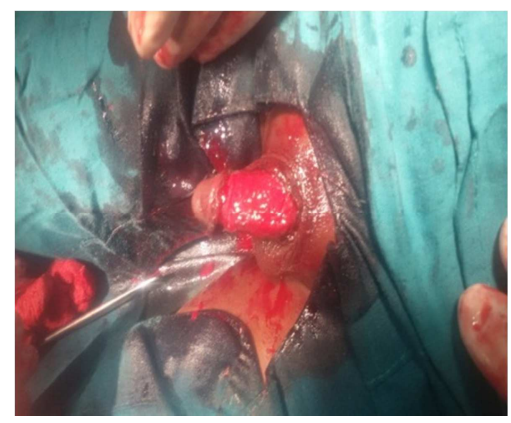

Figure 4. Nut removed after completely degloving penile skin

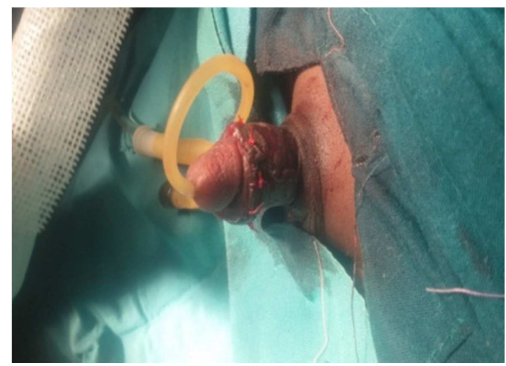

Figure 5. Penile skin reconstructed and catheter inserted.

Postoperatively he was placed on intravenous fluid, antibiotics and analgesic. He did well and the skin healed well over the phallus within two weeks (Fig. 6). He was however lost to follow up.

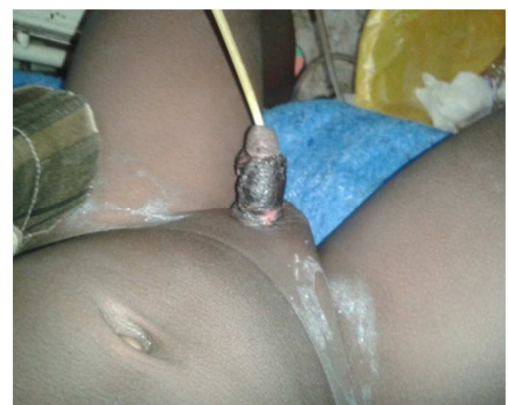

Figure 6. Two weeks after surgery, wound has healed.

\section{Discussion}

Penile constricting injury is a true urologic emergency as the intervention time to remove the constricting object determines if the penis could be salvaged [5]. This is especially where patient present early before the onset of ischaemia. The presentation often is late because a lot of the patients have either psychiatric illness or are children who are 
scared to mention it to parents for fear of punishment.

Penile strangulation is an uncommon clinical condition that was first reported in 1755 by Gauthier. Since then, various degrees of penile constriction injuries have been reported with different types of objects being used and various methods of their removal [2].

Children who have poor parental supervision are at greater risk of innocent childish experimentation of penile constricting object as in the index case. Non-metallic objects such as plastic encircling agent, hair and thread are predominantly used to control enuresis/urinary incontinence [6].

The injury may affect one or more tissues of the penis or the urethra. As the corpus spongiosum and urethra are covered by a thin layer of fibrous tissue they are most susceptible to injury. However, gangrene is uncommon, probably because each corpus cavernosum has an individual artery, and the thickness of Buck's fascia and corporeal tissue resists pressure on the deep vessels [2].

AL Bhat et al (1999) graded these injuries as follows [2, 5]:

Grade I Oedema of distal penis. No evidence of skin ulceration or urethral injury.

Grade II Injury to skin and constriction of corpus spongiosum but no evidence of urethral injury distal penile oedema with decreased penile sensation.

Grade III Injury to skin and urethra but no urethral fistula. Loss of distal penile sensations.

Grade IV Complete division of Corpus spongiosum leading to urethral fistula and constriction of corpus cavernosa with loss of distal penile sensations.

Grade $V$ Gangrene, necrosis, or complete amputation of distal penis.

The index case we presented had grade II injury using the above grading system. In our patient there was obvious constriction of the skin and oedema of the distal part of the penis. The difficulty in passing urine suggested constriction of the corpus spongiosum and the urethra. However, there was no injury to the urethra. Loss of distal penile sensation could not be established as the patient was lost to follow up.

Most cases of penile constriction injuries are acute in nature, however, there can be chronic form usually caused by inappropriate usage of devices developed for autoerotic purposes and to prolong erection [3].

Diagnosis is clinically obvious; in some form of the penile constriction diagnostic tools can be used to determine the extent of the injury. These include Doppler ultrasound to assess the blood flow and urethrogram to rule out urethral involvement [3,5].

The main objective in the treatment of penile constriction injury is decompression by removing the constricting object as soon as possible to facilitate free blood flow and ease micturition. In the case of chronic penile constriction, treatment also involve educating the patient on the correct usage of erection devices [1]. There is no standard protocol for treatment of cases of penile constriction injury. Every case needs an individual approach depending upon the circumstances and facilities available. Some of the methods include aspiration of corpora or the use of saws, grinders, bolt cutters, drills and degloving of penile skin. Multidisciplinary approach with other disciplines such as plastic surgeon, psychiatrist and mechanics may be needed in some forms of the constriction $[7,8]$. Use of multiple options as in the index case may be tried in selected patients. If removal of the strangulating object is not possible by any of the methods because of the size of the object and/or an extreme degree of oedema, the penis may be denuded and postoperatively is treated with the same principles of severe traumatic penile and scrotal avulsion, including skin grafting or using a myo-fascial flap $[9,10]$.

\section{Conclusion}

Penile constricting injury by different types of objects is a rare urological emergency often presenting a serious challenge to the urologist. Early presentation and prompt treatment with successful decompression of the penis is needed to prevent potential complications of gangrene and loss of the penis. Greater supervision of children by parents and caregivers will prevent occurrence of the type of penile constriction we reported or at least to detect and remove it early.

\section{References}

[1] C Avinash, W David, F Melissa. Removal of a Penile Constriction Device with a Large Orthopaedic Pin Cutter. Case Rep Urol. 2014; 2014: 347-350.

[2] S Jain, A Gupta, T Singh, et'al. Penile strangulation by a hard plastic bottle: A case report. Indian Journal of Surgery. 2004; 66: 173-175.

[3] O Ivanovski, O Stankov, M Kuzmanoski, et'al. Penile Strangulation: Two Case Reports and Review of the Literature. J Sex Med. 2007; 4: 1775-1780.

[4] JC Orakwe, TU Mbaeri, JA Abiahu, et'al. Strangulating Penile Ring: A Case Report. Afrimedic Journal 2011; 2: 28-31.

[5] A Nuhu, S Edino, G Agbese, et'al. Penile Gangrene Due To Strangulation by a Metallic Nut: A Case Report and Review of the Literature. The Internet Journal of Surgery. 2008; 21.

[6] Z Nazir, K Rasheed, F Moazam. Penile Constrictive Band Injury. JPMA. 1993; 43: 137-139.

[7] P Chih, C Wang, C Chen. Penile Strangulation Due to a Metal Ring - A Case Report. Incont Pelvic Floor Dysfunct 2013; 7(2): 31.

[8] R A Talib, O Canguven, A Al Ansari. Treatment of penile strangulation by the rotating saw and 4-needle aspiration method: Two case reports. Archivio Italiano di Urologia e Andrologia 2014; 86: 2-3.

[9] S V Perovic. Severe Penile Injuries: Etiology, Management and Outcomes. Urologia Polska.2005; 58: 1-3.

[10] O A Raheem, H S Mirheydar, N D Patel, et'al. Surgical Management of Traumatic Penile Amputation: A Case Report and Review of the World Literature. Sexual Medicine. 2015; 10: 1-3. 